\title{
Sex differences in complex regional pain syndrome type I (CRPS-I) in mice
}

This article was published in the following Dove Press journal:

Journal of Pain Research

31 July 2017

Number of times this article has been viewed

\section{Chaoliang Tang \\ Juan $\mathrm{Li}^{2}$ \\ Wai Lydia Tai ${ }^{3}$ \\ Weifeng Yao ${ }^{4}$ \\ Bo Zhao' \\ Junmou Hong ${ }^{5}$}

Si Shi ${ }^{1}$

Song Wang ${ }^{2}$

Zhongyuan Xia'

'Department of Anesthesiology,

Renmin Hospital of Wuhan University,

Wuhan, Hubei Province, ${ }^{2}$ Department of Anesthesiology, Anhui Provincial Hospital of Anhui Medical University, Hefei, Anhui Provence, ${ }^{3}$ Department of Anaesthesiology, The University of Hong Kong, Hong Kong SAR, ${ }^{4}$ Department of Anesthesiology, The Third Affiliated Hospital of Sun Yat-sen University, Guangzhou, Guangdong Province, ${ }^{5}$ Department of Cardiovascular Surgery, Renmin Hospital of Wuhan University, Wuhan, Hubei Province, China
Correspondence: Zhongyuan Xia Department of Anesthesiology, Renmin Hospital of Wuhan University, 99 Zhang road, Wuhan, Hubei 430060, China $\mathrm{Tel}+86$ I380862 8560

$\mathrm{Fax}+86278804$ । 911

Email xiazhongyuan2005@aliyun.com
Background: Sex differences have been increasingly highlighted in complex regional pain syndrome (CRPS) in clinical practice. In CRPS type I (CRPS-I), although inflammation and oxidative stress have been implicated in its pathogenesis, whether pain behavior and the underlying mechanism are sex-specific is unclear. In the present study, we sought to explore whether sex differences have an impact on inflammation, oxidative stress, and pain sensitivity in CRPS-I. Methods: Chronic post-ischemia pain (CPIP) was established in both male and female mice as an animal model of CRPS-I. Edema and mechanical allodynia of bilateral hind paws were assessed after reperfusion. Blood samples were analyzed for serum levels of oxidative stress markers and inflammatory cytokines.

Results: Both male and female mice developed edema. Male mice developed CPIP at day 3 after reperfusion; female mice developed CPIP at day 2 after reperfusion. Female mice displayed significantly earlier and higher mechanical allodynia in the ischemic hind paw, which was associated with higher serum levels of IL-2, TNF- $\alpha$, isoprostanes, 8 OhdG, and malondialdehyde at day 2 after reperfusion. Moreover, female mice showed significantly lower SOD and IL-4 compared to male mice at day 2 after reperfusion.

Conclusion: Our results indicate that sex differences in inflammatory and oxidative stress states may play a central role in the sex-specific nociceptive hypersensitivity in CRPS-I, and offer a new insight into pharmacology treatments to improve pain management with CRPS.

Keywords: sex differences, complex regional pain syndrome, chronic post-ischemia pain, inflammatory response, oxidative stress

\section{Introduction}

Complex regional pain syndrome (CRPS) is a common disabling pain condition that can develop after fractures, limb trauma, or lesions of the peripheral or the central nervous system. ${ }^{1}$ CRPS is classified into two types: CRPS-I (without major nerve damage) and CRPS-II (with nerve injury). ${ }^{2}$ The pathogenesis of CRPS remains largely unknown, rendering this syndrome a global challenge in both diagnosis and treatment. Clinically, CRPS is characterized by neuropathic pain, as well as sensory-motor and autonomic symptoms. ${ }^{3}$ In recent decades, an animal model of CRPS was established, namely the chronic post-ischemia pain (CPIP) model, which produces symptoms highly identified as the essential features of CRPS-I in humans. ${ }^{4}$ This model enabled in-depth mechanistic studies of the pathogenesis of CRPS, in which inflammatory cytokines, such as IL-2, TNF- $\alpha$, and IL-4, and oxidative stress were recognized as major contributors. ${ }^{3,5,6}$

Evidence indicates that the primary mechanisms governing the emergence of CRPS symptoms include ischemia, trauma-related cytokine release, inflammation, and oxidative 
stress in response to chronic pain. ${ }^{7-9}$ However, these results are concluded from studies using only male animals. While sex differences in pain sensitivity have demonstrated increasing importance in both clinical and experimental studies conducted in the past few decades, the results remain hotly debated. ${ }^{10-12}$ Some studies showed notable sex differences in pain sensitivity whereas others did not. Nonetheless, the majority of patients with chronic pain are female, and some evidence suggests that females may be more vulnerable to central sensitization initiated by peripheral inflammation. ${ }^{13,14}$ It is possible that a higher incidence of anxiety disorders and/ or lower pain tolerance in females contributes to pain experienced earlier than in males, leading to higher percentages of females crossing the threshold at which experienced pain rises to the level of a diagnosed "pain syndrome". ${ }^{15}$

Apart from the sex differences in the onset of nociceptive disorder, one hypothesis is that females may also have a higher inflammatory state in chronic pain. As inflammation plays a critical role in CRPS, sex differences may have an important implication in its pathogenesis. However, no study has compared inflammatory markers between female and male animals in the CPIP model. Thus, characterizing sex differences in CPIP inflammatory markers may provide insight into sex differences in long-term outcomes and potential sex-specific treatments of CRPS-I. We hypothesized that higher levels of inflammatory markers after CPIP in female mice than in male mice may account for prolonged nociception in female mice in CRPS-I. Findings from this study may enhance our understanding of the contribution of inflammatory markers to sex differences in nociceptive hypersensitivity in CRPS-I.

\section{Materials and methods Animals}

Animal experiments were carried out according to the US National Institutes of Health Guide for the Care and Use of Laboratory Animals, and approved by Institutional Animal Care and Use Committee of Wuhan University. In this study, adult male and female C57BL/6 wild-type mice (28-30 g) were obtained from the Laboratory Animal Unit of Wuhan University. Mice were housed at $23 \pm 3^{\circ} \mathrm{C}$, with a 12-hour light/dark cycle (lights on at 07:00) and humidity (25\%-45\%). Animals were offered free access to water and food (Lab Diet 5012 [0.5\% phosphorus, $1.0 \%$ calcium and $3.3 \mathrm{IU} / \mathrm{g}$ of vitamin D3]).

\section{CPIP model}

The CPIP procedure was performed from approximately 13:00 to $17: 00$. Mice were initially anesthetized with intraperitoneal pentobarbital $55 \mathrm{mg} / \mathrm{kg}$, followed by $27.5 \mathrm{mg} / \mathrm{kg}$ when required over a $3 \mathrm{~h}$ period. After induction of anesthesia, a Nitrile 70 Durometer O-ring with a 5/64-inch internal diameter was placed around the right ankle joint of mice for $3 \mathrm{~h} .{ }^{16}$ In the third hour, the O-ring was cut and reperfusion occurred. The termination of pentobarbital anesthesia was timed so that the mice recovered fully within $30-60$ min following reperfusion.

\section{CPIP criteria}

Successful development of CPIP relies on the existence of mechanical allodynia, which meets the criteria of a $30 \%$ decrease in the mechanical threshold of an ischemic limb (ipsilateral side) at about $48 \mathrm{~h}$ after reperfusion. ${ }^{4}$ Animals fulfilling the criteria would be regarded as having successfully developed CPIP.

\section{Hind paw volume}

The volume of the mouse hind paw was measured with the U-shaped volume sensor (IITC Life Science Inc., Woodland Hills, CA, USA) following the manufacturer's protocol. In use, the paw was inserted into plain water contained within a water cell, thereby changing the volume due to the immersion. This volume change was calibrated in $\mathrm{mL}$ and was displayed on an electronic monitor.

\section{Mechanical allodynia}

The mice were taken to the laboratory platform for $1 \mathrm{~h}$ per day for a total of 2 days prior to the experiment and $30 \mathrm{~min}$ before behavior assessment on the experiment day to become accustomed to the laboratory environment. The investigator assessing the physical signs and pain behavior of the mice was blinded to the study groups.

The paw withdrawal threshold (PWT) of mice was assessed by the von Frey test with a von Frey fiber of an Electrovonfrey apparatus (IITC Life Science Inc.) as described in our previous study. ${ }^{8}$ The von Frey testing was performed from approximately 09:00 to 12:00. Briefly, mice were placed on a metal mesh floor with a transparent plastic dome for about 30 min before the experiment. During the assessment, a series of von Frey filaments (IITC) with stiffness ranging from $0.008 \mathrm{~g}$ to $6 \mathrm{~g}$ was applied to the plantar surface of hind paws respectively. Each filament was applied for $4 \mathrm{~s}$ or sufficient to bend the filaments into an " $\mathrm{S}$ " shape. If the mouse withdrew the hind paw from the filament, the displayed value of this force on the apparatus was taken as the PWT of the mouse. If no response was observed, a stiffer probe was used. The withdrawal threshold was measured at 5 to 10 min intervals, alternating between the left and right sides until each side had been tested three times. PWT was 
measured before ischemia (baseline) and from day 1 up to day 28 after reperfusion.

\section{Serum oxidative stress biomarkers and inflammatory cytokines}

Oxidative stress and inflammatory responses can indicate the involvement of the inflammatory process in CPIP. As the earliest start time for female mice to develop mechanical allodynia was 2 days post-reperfusion and the difference of betweengroup comparison persisted up to 7 days post-reperfusion in the CPIP model, we chose 2 and 7 days post-reperfusion as the time-points to ensure mechanistic exploration. Hence, blood samples were collected at baseline, 2 days after reperfusion, and 7 days after reperfusion, and stored overnight at $4^{\circ} \mathrm{C}$. Samples were then centrifuged at $4000 \mathrm{rpm}$ for $10 \mathrm{~min}$ and supernatant was taken and allocated for assessment. Serum isoprostanes, 8 OhdG, malondialdehyde (MDA), IL-2, TNF$\alpha$, and IL- 4 were measured according to the manufacturer's instructions using enzyme-linked immunosorbent assay (ELISA) methods (Cloud-Clone Corp., Houston, TX, USA). The activity of SOD was determined using a commercial kit (Nanjing Jiancheng Bioengineering Institute, Nanjing, China) as previously described. ${ }^{17}$

\section{Statistical analysis}

All data were presented as means \pm standard error of the mean (SEM). Edema and PWT data were analyzed with two-way repeated measures analysis of variance (ANOVA) with a Greenhouse-Geisser correction for sphericity in a manner that ensured the data were compared on a complete study time-course basis instead of individual time-points. Serum oxidative stress markers and inflammatory cytokines were evaluated using a Mann-Whitney $U$ test for within-group comparisons and a Wilcoxon signed rank test for betweengroup comparisons. Statistical analysis was performed with the SPSS 22.0 statistical software package (IBM Corporation, Armonk, NY, USA) in line with the intention-to-treat principle. $P<0.05$ was considered statistically significant.

\section{Results CPIP-induced edema volume is not sex- specific}

Contralateral hind paws of both sexes exhibited no statistical difference before and after CPIP (Figure 1A and B). CPIP injury significantly increased the volume of ipsilateral hind paws of both male and female mice from $1 \mathrm{~h}$ to 2 days post-reperfusion compared to their contralateral hind paws, respectively $(P<0.05$, $P<0.001$; ipsilateral vs contralateral; two-way ANOVA, $F[1$,
$60]=508.9$, and $F[1,60]=680.7$, respectively; $\mathrm{n}=6$ ) (Figure $1 \mathrm{~A}$ and $\mathrm{B})$. The results indicated successful establishment of the CPIP model in both male and female mice. However, no statistical difference was found between sexes in both ipsilateral and contralateral hind paws (Figure 1C and D).

\section{Female mice display earlier onset and higher intensity of mechanical allodynia than male mice in CRPS-I}

As shown in Figure 2A and B, CPIP induced mechanical allodynia on ipsilateral sides only, in both sexes. Earlier onset of mechanical allodynia in females was demonstrated by significantly reduced ipsilateral PWT from 2 to 14 days post-reperfusion in female mice as compared to that from 3 to 14 days post-reperfusion in male mice $(P<0.05, P<0.01$, and $P<0.001$; ipsilateral vs contralateral; two-way ANOVA, $F[1,460]=765.0$, and $F[1,460]=493.7$, respectively; $\mathrm{n}=$ $8)$. No statistical difference was detected on the contralateral sides between male and female mice (Figure 2C). However, on the ipsilateral side, females displayed significantly lower PWT than that of males from 1 to 7 days post-reperfusion $(P<0.05, P<0.01$, and $P<0.001$; male vs female; two-way ANOVA, $F[1,460]=159.3 ; \mathrm{n}=8)$ (Figure 2D).

\section{Oxidative stress levels are sex-specific during the onset of CRPS-I}

Serum levels of isoprostanes, 8 OhdG, and MDA (biomarkers of oxidative stress) were increased while SOD (antioxidant) was decreased significantly in both sexes on day 2 after CPIP compared to their baseline, respectively $(P<0.05$ vs baseline; $\mathrm{n}=8$ ) (Figure 3). No significant difference was detected between post-reperfusion day 7 and baseline levels of each oxidative stress parameter in both sexes, except for SOD levels in female mice $(P<0.05$ vs baseline; $\mathrm{n}=8)$ (Figure 3D). In a comparison between sexes, we found significantly higher levels of isoprostanes, 8 OhdG, and MDA in female mice than that of male mice on day 2 post-reperfusion $(P<0.05$; male vs female; $\mathrm{n}=8$ ) (Figure $3 \mathrm{~A}$ to $\mathrm{C}$ ). Furthermore, SOD level was significantly lower in females compared to that of males $(P<0.05$; male vs female; $\mathrm{n}=8)$ (Figure 3D). However, no significant difference of the serum oxidative stress markers was observed on post-reperfusion day 7 between sexes.

\section{Inflammatory cytokines are expressed differently between sexes during initiation of CRPS-I}

As demonstrated in Figure 4A, the serum concentration of IL-2 was increased significantly on day 2 and 7 post-reperfusion 
A

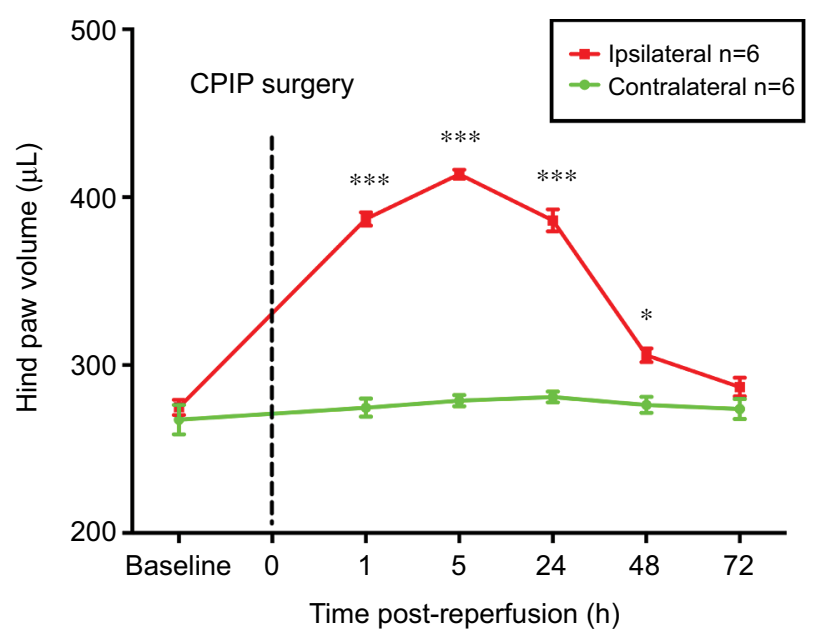

C

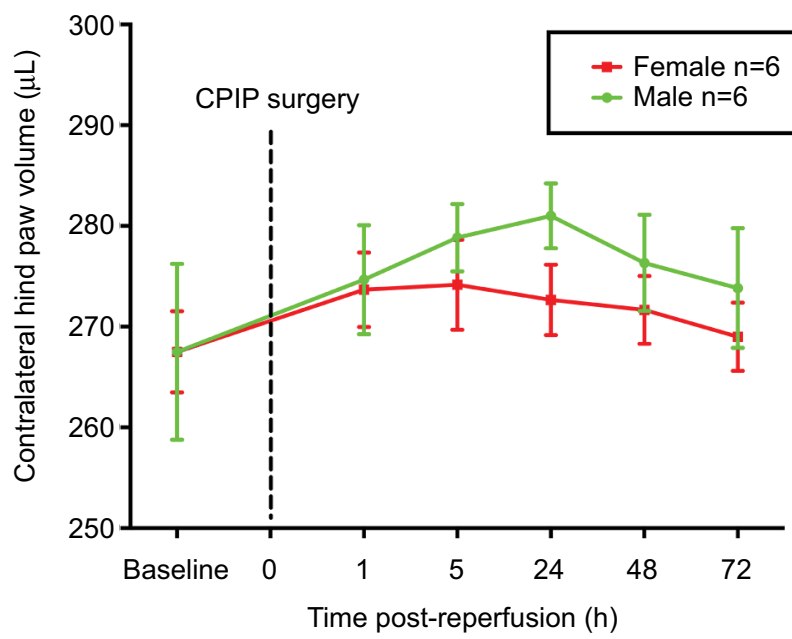

B
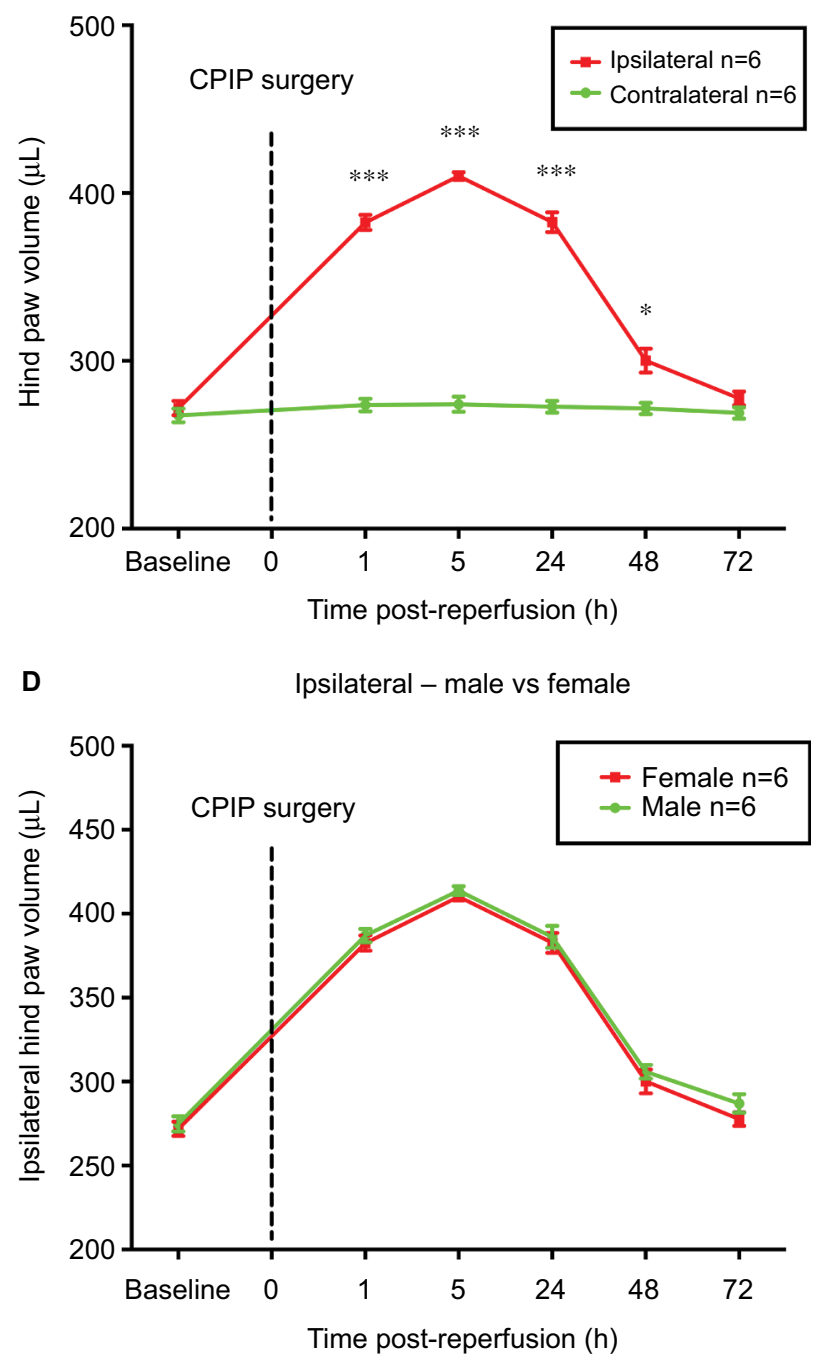

Figure I Hind paw volumes of male and female mice after CPIP.

Notes: Effects of CPIP injury on the volume of the contralateral and ipsilateral hind paws of male (A) and female mice (B). Volume of the contralateral hind paws was compared between male and female $(\mathbf{C})$ as well as the ipsilateral hind paws $(\mathbf{D})$ after CPIP surgery. Results are presented as means $\pm s t a n d a r d$ error of the mean. $* P<0.05, * * * P<0.00 I ; n=6$. Abbreviation: CPIP, chronic post-ischemia pain.

in both sexes $(P<0.001$ vs baseline; $\mathrm{n}=8)$. IL-2 level was only significantly higher in female mice than that in male mice at 2 days after CPIP $(P<0.01$; male vs female; $\mathrm{n}=8)$. In Figure 4B, CPIP increased TNF- $\alpha$ levels significantly in both sexes on day 2 post-reperfusion $(P<0.001$ vs baseline; $\mathrm{n}=8$ ), and TNF- $\alpha$ level was higher in females than in males $(P<0.05$; male vs female; $\mathrm{n}=8)$. Unlike IL-2 and TNF- $\alpha$, which are pro-inflammatory cytokines, IL-4 (Figure 4C) is an anti-inflammatory cytokine and was decreased on both day 2 and 7 post-reperfusion in females $(P<0.001$ and $P<0.05$, respectively vs baseline; $\mathrm{n}=8$ ) while it was only decreased on day 2 post-reperfusion in male mice $(P<0.001$ vs baseline; $\mathrm{n}=8$ ). Furthermore, IL-4 level was significantly lower in female than in male mice at 2 days post-reperfusion $(P<0.01$; male vs female; $\mathrm{n}=8$ ).

\section{Discussion}

Here, we reproduced a CPIP model in both male and female mice by inducing CRPS-I-like symptoms such as edema and mechanical allodynia to study whether sex differences are present in CRPS-I. We found that female mice displayed earlier onset and greater degree of mechanical allodynia than that of male mice in CRPS-I. In addition, a higher state of inflammation and oxidative stress was found in females than in males during the initiation of CRPS-I, which may account for the sex-specific nociceptive behavior of female mice after CPIP.

Clinical trials have pointed out the pathophysiological role of cytokines in the induction and maintenance of pain in patients with CRPS. ${ }^{18}$ Persistent inflammation after ischemia-reperfusion injury may be a crucial factor in the 


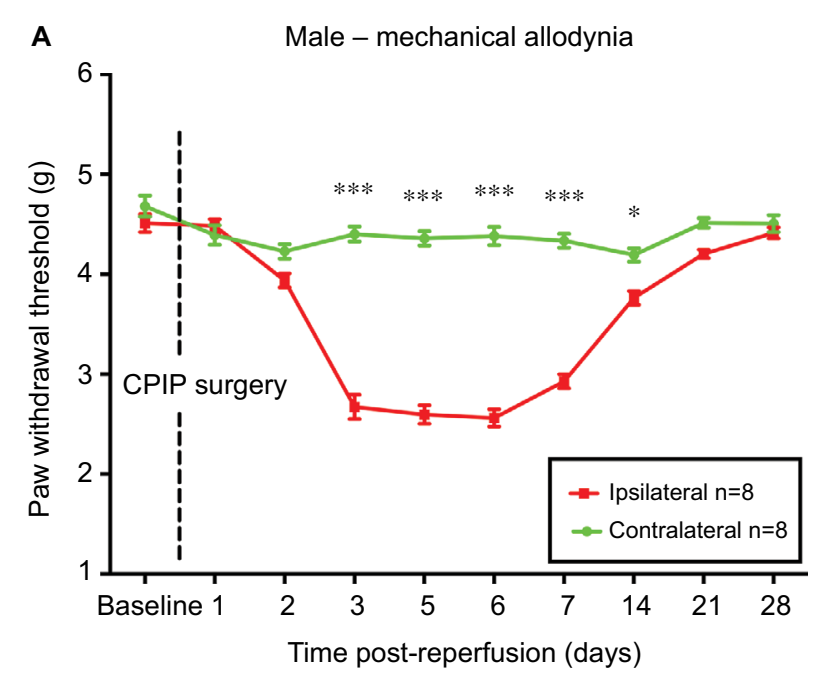

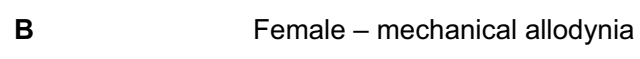
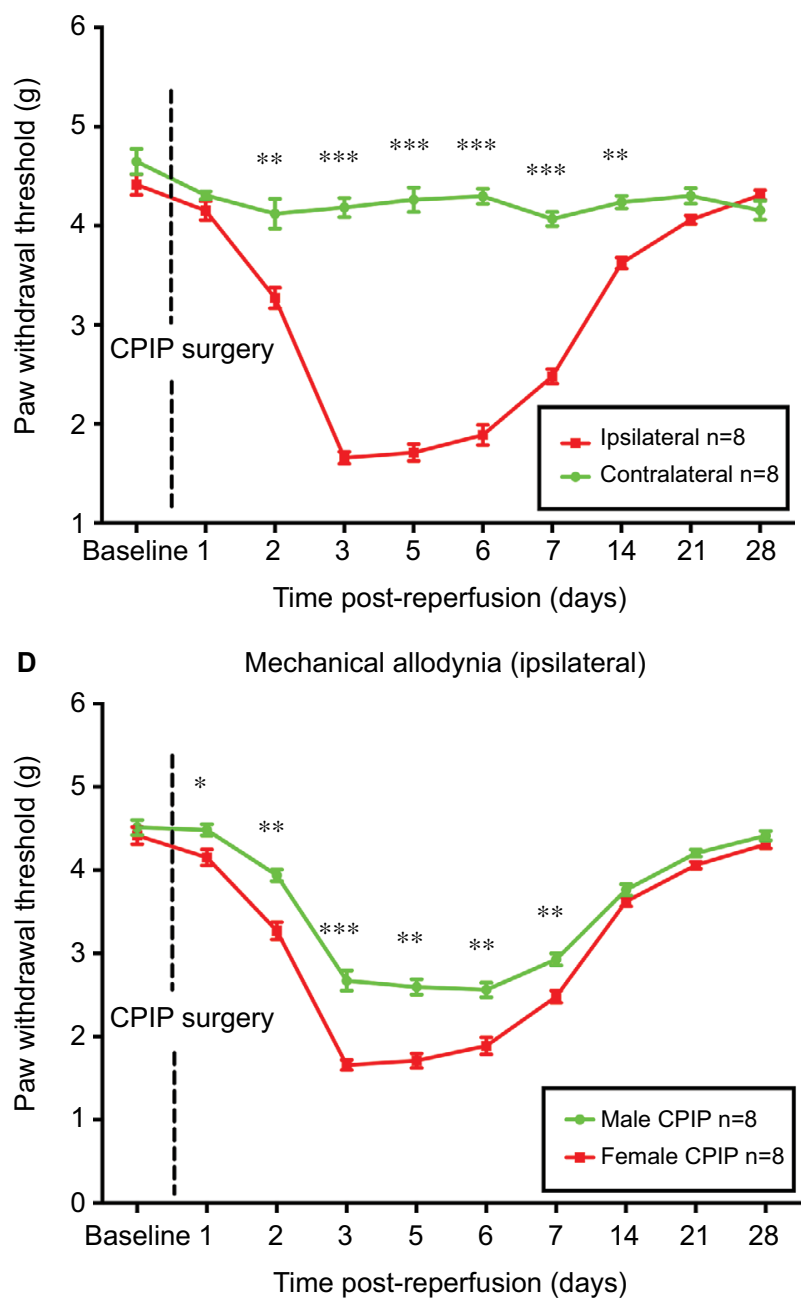

Figure 2 Paw withdrawal threshold of male and female mice after CPIP.

Notes: Paw withdrawal threshold of the contralateral and ipsilateral sides of male (A) and female mice (B) was monitored for 28 days after CPIP surgery. Paw withdrawal threshold of the contralateral hind paws was compared between males and females (C) as well as the ipsilateral hind paws (D). Results are presented as means \pm standard error of the mean. $* P<0.05, * * P<0.01, * * *<0.001 ; \mathrm{n}=8$.

Abbreviation: CPIP, chronic post-ischemia pain.

pathogenesis of CPIP. ${ }^{4,18,19}$ While pro-inflammatory cytokines are predominantly algesic, anti-inflammatory cytokines show mostly analgesic effects. ${ }^{18,20}$ Studies have shown that pro-inflammatory cytokine TNF- $\alpha$ induces pain after local application and is upregulated in pain models while upregulation of IL-4 or IL-10 produces analgesic effects. ${ }^{21,22}$ Indeed, in the present study, serum levels of pro-inflammatory cytokines IL-2 and TNF- $\alpha$ were significantly elevated and anti-inflammatory cytokine IL-4 was reduced after CPIP in both sexes, suggesting inflammation may be a key contributor to CRPS in female mice as in male mice.

Yet, the pathophysiology of sex differences in CRPS is still incompletely understood. Previous evidence of sex differences in inflammatory markers showed that females often present greater levels of pro-inflammatory markers following immune challenge. ${ }^{23,24}$ Some animal research has related this phenomenon to the higher peripheral and central T-cell counts in female than in male mice. ${ }^{25,26}$ Additionally, infiltrated T-cells in nerve-injured female mice may secrete more pro-inflammatory markers relative to male mice, ${ }^{27}$ such as TNF- $\alpha$ and IL-2, or protective/trophic factors like IL-4. ${ }^{28}$ Hence after ischemia-reperfusion injury, levels of TNF- $\alpha$ and IL- 2 are rapidly upregulated. ${ }^{7}$ Our finding is in support of the previously mentioned notion that female mice have higher serum levels of pro-inflammatory cytokines IL-2 and TNF- $\alpha$, and lower serum levels of antiinflammatory cytokines IL-4 after ischemia-reperfusion injury, suggesting that inflammation has a critical role in the sex differences in CRPS. Taken together, we suggest that the immune system in female mice may release more pro-inflammatory cytokines such as IL-2 and TNF- $\alpha$, and less anti-inflammatory cytokines such as IL-4 to 
A

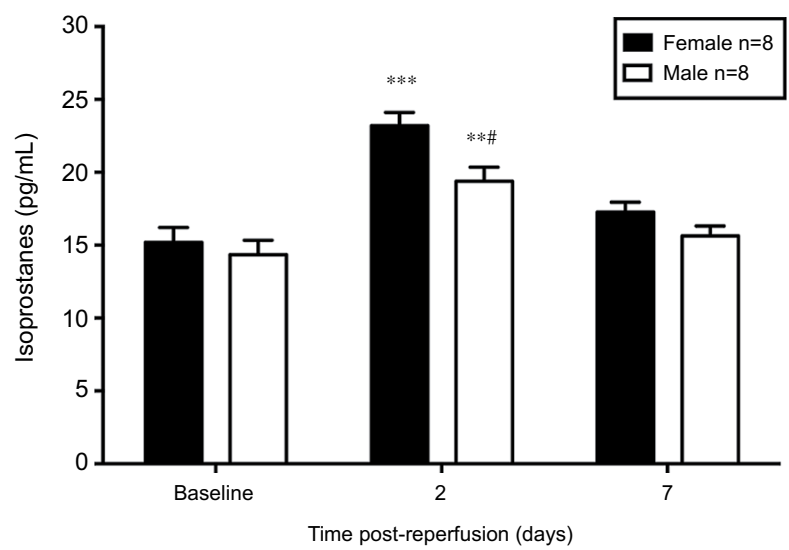

C

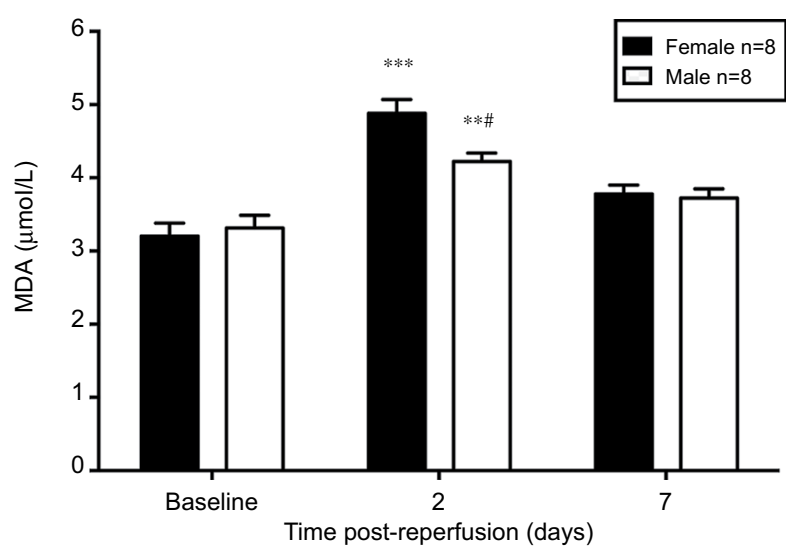

B

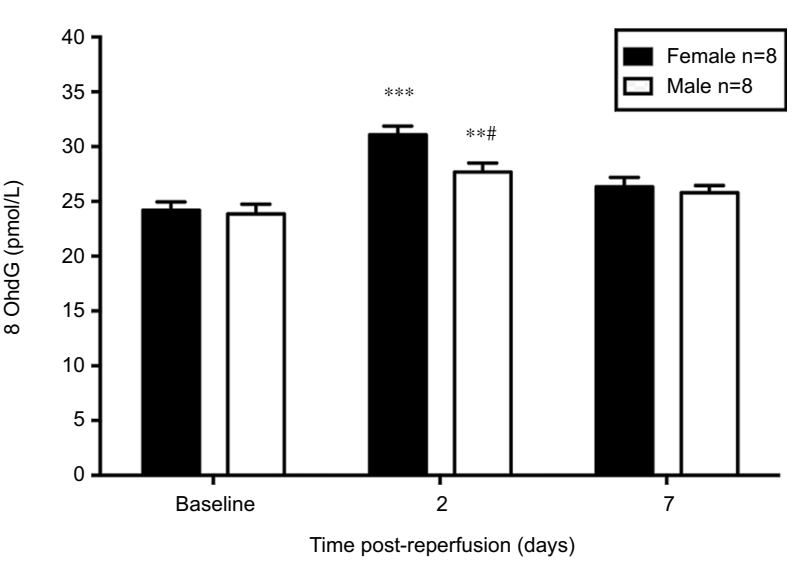

D

Serum SOD

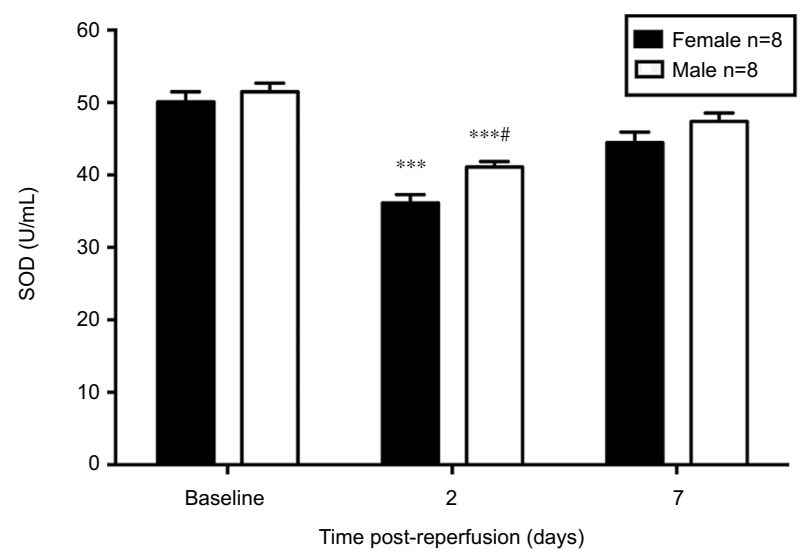

Figure 3 Serum level of oxidative stress biomarkers in male and female mice after CPIP.

Notes: Blood serum was collected at baseline, 2 days, and 7 days post-reperfusion to assess the serum level of isoprostanes (A), 8 OhdG (B), MDA (C), and SOD (D). Results are presented as means \pm standard error of the mean. $* P<0.05, * * P<0.01$ and $* * * P<0.001$ vs baseline; ${ }^{*} P<0.05$, male vs female; $n=8$.

Abbreviations: CPIP, chronic post-ischemia pain; MDA, malondialdehyde.

enhance inflammation and sensitize afferent nociceptors after ischemia-reperfusion injury, which leads to a greater nociceptive hypersensitivity in female mice in CRPS. Our results may inspire further studies to examine the potential sex differences in T-cell activities in the pain process, and perhaps T-cells may offer a new insight into pharmacology treatments to improve pain management in females and males with CRPS.

In addition to inflammation, oxidative stress is also a crucial contributor to the pathogenesis of CRPS. Previous studies demonstrated that ischemia-reperfusion injury-induced chronic pain is mediated by superoxide and nitric oxide. Administration of reactive oxygen species scavenger SOD during the peri-reperfusion period had long-term analgesic effects in CPIP. ${ }^{6}$ Also, tissue injury and inflammation often result in an oxidative burst, accumulation of hydrogen peroxide and hydroxyl radicals at the sites of trauma, ${ }^{29}$ and one might expect enhanced lipid peroxidation and thus the formation of isoprostanes. ${ }^{30}$ Furthermore, isoprostanes could lower nociceptive thresholds to mechanical and thermal stimuli. In theory, the release of reactive oxygen species can give rise to a greater level of inflammatory mediators evidenced by higher concentrations of local and circulating cytokines. ${ }^{31,32}$ In the present study, investigation of oxidative stress parameters revealed that serum concentrations of isoprostanes, 8 OhdG, and MDA were significantly increased while SOD was decreased significantly in both sexes at the initiation of CRPS-I. In particular, female mice showed significantly higher degrees of these alterations than males, suggesting a greater state of oxidative stress in female mice during CPIP.

Although clinical research usually recruits both males and females, female animals remain a less popular choice of research subject in experimental studies. The omission of female animals from preclinical experiments can have serious implications, as some sex differences are qualitative rather than quantitative, and failure to appreciate them can lead to either missing biological phenomena entirely or overgeneralization of findings. As we know, estrogens are widely 
A

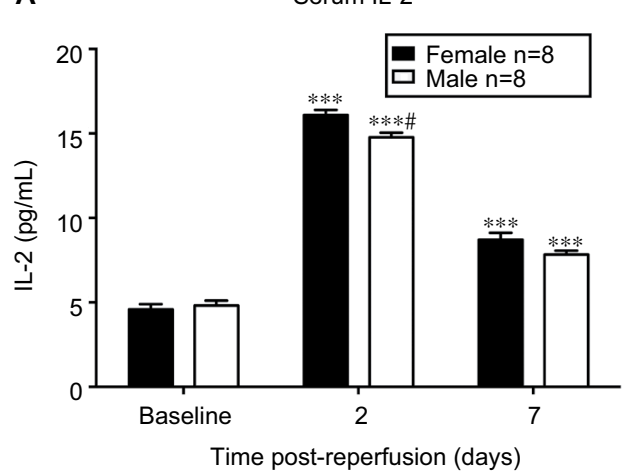

B

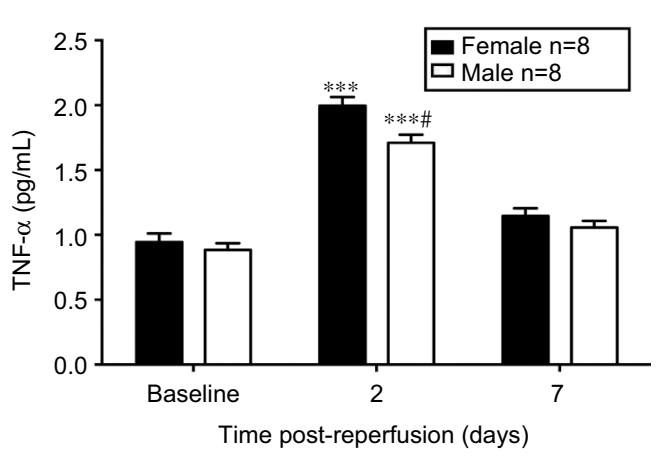

C

Serum IL-4

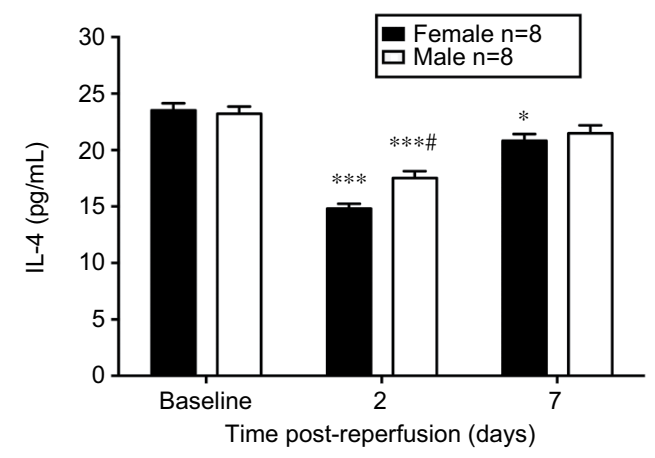

Figure 4 Serum level of inflammatory cytokines in male and female mice after CPIP.

Notes: Serum levels of IL-2 (A), TNF- $\alpha(\mathbf{B})$, and IL-4 (C) were assessed at baseline, 2 days post-reperfusion, and 7 days post-reperfusion. Results are presented as means \pm standard error of the mean. $* P<0.05$ and $* * *<0.001$ vs baseline; ${ }^{*} P<0.05$ male vs female; $\mathrm{n}=8$.

Abbreviation: CPIP, chronic post-ischemia pain.

involved in mechanisms of inflammation; ${ }^{33,34}$ for example, estrogens influence the metabolism of protein substance $P$ (SP) and bradykinin and the formation of reactive oxygen species during ischemia or inflammation. ${ }^{35,36}$ Moreover, $\mathrm{NF}-\mathrm{kB}$, an important transcription factor in inflammation could be mediated by interaction with estrogen receptors. ${ }^{37,38}$ In support, the current study has demonstrated higher serum levels of pro-inflammatory cytokines IL-2 and TNF- $\alpha$ in female than in male mice in the development of CRPS-I. In fact, in experimental pain models, estrogen was found to increase brain-derived neurotrophic factor-mediated neuropathic and nociceptive pain, suggesting a hypothetical downstream mechanism of action in the pathogenesis of CRPS. ${ }^{39,40}$ Like androgen receptors, estrogen receptors are also present in the central nervous system. In the brain, there is a high concentration of these receptors in the hypothalamus and in parts of the limbic forebrain thought to regulate homeostasis, reproduction, emotional behavior, and pain. ${ }^{41}$ Some researchers indicated that male gonadal hormones acting at central levels decrease the ability of the central nervous system to retain information, including information related to nociceptive input, and then inhibit the behavioral response to repeated nociceptive stimulation. ${ }^{42}$ This evidence suggests that male gonadal hormones have an antinociceptive role while estrogens have a pronociceptive role, which could explain, at least in part, the higher incidence of chronic pain syndromes in females.

In conclusion, we demonstrated for the first time that nociceptive hypersensitivity, inflammation, and oxidative stress states are sex-specific in CRPS-I. The study will not attempt to comprehensively review the underlying mechanisms, but will attempt to illustrate a phenomenon as well as the preliminary explanations. The underlying sex differences in inflammation are likely to impact ischemia-reperfusion injury-evoked chronic pain in CPIP, and potentially contribute to the differential behavioral outcome of ischemiareperfusion injury in male and female mice. Ultimately, this study provides a novel understanding of sex differences in the pain process and new insight into pharmacology treatments to improve pain management in male and female CRPS patients.

\section{Acknowledgments}

The authors are grateful for the enthusiastic support of $\mathrm{Mr}$ Kai Zhang in creating the figures. This study was supported by the National Natural Science Foundation of China (grant no 81671891). 


\section{Disclosure}

The authors report no conflicts of interest in this work.

\section{References}

1. Wang L, Guo TZ, Wei T, Li WW, Shi X, Clark JD, Kingery WS. Bisphosphonates inhibit pain, bone loss, and inflammation in a rat tibia fracture model of complex regional pain syndrome. Anesth Analg. 2016;123(4):1033-1045.

2. Borchers AT, Gershwin ME. Complex regional pain syndrome: a comprehensive and critical review. Autoimmun Rev. 2014;13(3):242-265.

3. Bussa M, Guttilla D, Lucia M, Mascaro A, Rinaldi S. Complex regional pain syndrome type I: a comprehensive review. Acta Anaesthesiol Scand. 2015;59(6):685-697.

4. Coderre TJ, Xanthos DN, Francis L, Bennett GJ. Chronic postischemia pain (CPIP): a novel animal model of complex regional pain syndrome-type I (CRPS-I; reflex sympathetic dystrophy) produced by prolonged hindpaw ischemia and reperfusion in the rat. Pain. 2004;112(1-2):94-105.

5. Schiller PW, Nguyen TM, Saray A, Poon AW, Laferriere A, Coderre TJ. The bifunctional $\mu$ opioid agonist/antioxidant [Dmt(1)]DALDA is a superior analgesic in an animal model of complex regional pain syndrome-type i. ACS Chem Neurosci. 2015;6(11):1789-1793.

6. Kwak KH, Han CG, Lee SH, et al. Reactive oxygen species in rats with chronic post-ischemia pain. Acta Anaesthesiol Scand. 2009;53(5): 648-656.

7. Liman S, Cheung CW, Wong KL, et al. Preventive treatment with ketamine attenuates the ischaemia-reperfusion response in a chronic postischaemia pain model. Oxid Med Cell Longev. 2015;2015:380403.

8. Tian G, Luo X, Tang C, et al. Astrocyte contributes to pain development via MMP2-JNK1/2 signaling in a mouse model of complex regional pain syndrome. Life Sci. 2017;170:64-71.

9. Wesseldijk F, Fekkes D, Huygen FJ, van de Heide-Mulder M, Zijlstra FJ. Increased plasma glutamate, glycine, and arginine levels in complex regional pain syndrome type 1. Acta Anaesthesiol Scand. 2008;52(5):688-694.

10. Tschugg A, Loscher WN, Lener S, Wildauer M, Hartmann S, Neururer S, Thomé C. Gender differences after lumbar sequestrectomy: a prospective clinical trial using quantitative sensory testing. Eur Spine $J$. 2017;26(3):857-864.

11. Kindler LL, Valencia C, Fillingim RB, George SZ. Sex differences in experimental and clinical pain sensitivity for patients with shoulder pain. Eur J Pain. 2011;15(2):118-123.

12. Boerner KE, Eccleston C, Chambers CT, Keogh E. Sex differences in the efficacy of psychological therapies for the management of chronic and recurrent pain in children and adolescents: a systematic review and meta-analysis. Pain. Epub 2016 Dec 15.

13. Moieni M, Irwin MR, Jevtic I, Olmstead R, Breen EC, Eisenberger NI. Sex differences in depressive and socioemotional responses to an inflammatory challenge: implications for sex differences in depression. Neuropsychopharmacology. 2015;40(7):1709-1716.

14. Bekhbat M, Neigh GN. Sex differences in the neuro-immune consequences of stress: focus on depression and anxiety. Brain Behav Immun. Epub 2017 Feb 16.

15. Mogil JS. Sex differences in pain and pain inhibition: multiple explanations of a controversial phenomenon. Nat Rev Neurosci. 2012; 13(12):859-866.

16. Millecamps M, Laferriere A, Ragavendran JV, Stone LS, Coderre TJ. Role of peripheral endothelin receptors in an animal model of complex regional pain syndrome type 1 (CRPS-I). Pain. 2010;151(1): 174-183.

17. Li H, Yao W, Liu Z, et al. Hyperglycemia abrogates ischemic postconditioning cardioprotection by impairing AdipoR1/Caveolin-3/STAT3 signaling in diabetic rats. Diabetes. 2016;65(4):942-955.

18. Alexander GM, Peterlin BL, Perreault MJ, Grothusen JR, Schwartzman RJ. Changes in plasma cytokines and their soluble receptors in complex regional pain syndrome. J Pain. 2012;13(1):10-20.
19. Uceyler N, Eberle T, Rolke R, Birklein F, Sommer C. Differential expression patterns of cytokines in complex regional pain syndrome. Pain. 2007;132(1-2):195-205.

20. Marchand F, Perretti M, McMahon SB. Role of the immune system in chronic pain. Nat Rev Neurosci. 2005;6(7):521-532.

21. Milligan ED, Sloane EM, Langer SJ, et al. Repeated intrathecal injections of plasmid DNA encoding interleukin-10 produce prolonged reversal of neuropathic pain. Pain. 2006;126(1-3):294-308.

22. Hao S, Mata M, Glorioso JC, Fink DJ. HSV-mediated expression of interleukin-4 in dorsal root ganglion neurons reduces neuropathic pain. Mol Pain. 2006;2:6.

23. Morrison HW, Filosa JA. Sex differences in astrocyte and microglia responses immediately following middle cerebral artery occlusion in adult mice. Neuroscience. 2016;339:85-99.

24. Bollinger JL, Bergeon Burns CM, Wellman CL. Differential effects of stress on microglial cell activation in male and female medial prefrontal cortex. Brain Behav Immun. 2016;52:88-97.

25. Sorge RE, Mapplebeck JC, Rosen S, et al. Different immune cells mediate mechanical pain hypersensitivity in male and female mice. Nat Neurosci. 2015;18(8):1081-1083.

26. Bordon Y. Neuroimmunology: a painful difference between the sexes. Nat Rev Immunol. 2015;15(8):469.

27. Mapplebeck JC, Beggs S, Salter MW. Molecules in pain and sex: a developing story. Mol Brain. 2017;10(1):9.

28. Beers DR, Henkel JS, Zhao W, et al. Endogenous regulatory T lymphocytes ameliorate amyotrophic lateral sclerosis in mice and correlate with disease progression in patients with amyotrophic lateral sclerosis. Brain. 2011;134(Pt 5):1293-1314.

29. Kehrer JP, Klotz LO. Free radicals and related reactive species as mediators of tissue injury and disease: implications for health. Crit Rev Toxicol. 2015;45(9):765-798.

30. Evans AR, Junger $\mathrm{H}$, Southall MD, et al. Isoprostanes, novel eicosanoids that produce nociception and sensitize rat sensory neurons. J Pharmacol Exp Ther. 2000;293(3):912-920.

31. Duflo F, Debon R, Goudable J, Chassard D, Allaouchiche B. Alveolar and serum oxidative stress in ventilator-associated pneumonia. $\mathrm{Br} J$ Anaesth. 2002;89(2):231-236.

32. Zhao P, Zhou WC, Li DL, et al. Total glucosides of Danggui Buxue Tang attenuate BLM-induced pulmonary fibrosis via regulating oxidative stress by inhibiting NOX4. Oxid Med Cell Longev. 2015;2015:645814.

33. Straub RH. The complex role of estrogens in inflammation. Endocr Rev. 2007;28(5):521-574.

34. Vasarhelyi B, Meszaros K, Karvaly G, Patocs A. Fókuszban a szöveti biomarkerek. Az ösztrogének mint a szövetspecifikus immunválasz és autoimmunitás modulálásának kulcsszereplöi [Focusing on tissue biomarkers. Estrogens as key players in the immune response and autoimmunity]. Orv Hetil. 2015;156(51):2070-2076. Hungarian.

35. Bjorling DE, Wang ZY. Estrogen and neuroinflammation. Urology. 2001;57(6 Suppl 1):40-46.

36. Murphy E, Steenbergen C. Gender-based differences in mechanisms of protection in myocardial ischemia-reperfusion injury. Cardiovasc Res. 2007;75(3):478-486.

37. Hettne KM, de Mos M, de Bruijn AG, et al. Applied information retrieval and multidisciplinary research: new mechanistic hypotheses in complex regional pain syndrome. J Biomed Discov Collab. 2007;2:2.

38. Kalaitzidis D, Gilmore TD. Transcription factor cross-talk: the estrogen receptor and NF-kappaB. Trends Endocrinol Metab. 2005;16(2):46-52.

39. Allen AL, McCarson KE. Estrogen increases nociception-evoked brain-derived neurotrophic factor gene expression in the female rat. Neuroendocrinology. 2005;81(3):193-199.

40. de Mos M, Huygen FJ, Stricker BH, Dieleman JP, Sturkenboom MC. Estrogens and the risk of complex regional pain syndrome (CRPS). Pharmacoepidemiol Drug Saf. 2009;18(1):44-52.

41. Manolagas SC, Kousteni S. Perspective: nonreproductive sites of action of reproductive hormones. Endocrinology. 2001;142(6):2200-2204.

42. Aloisi AM, Ceccarelli I, Fiorenzani P. Gonadectomy affects hormonal and behavioral responses to repetitive nociceptive stimulation in male rats. Ann NY Acad Sci. 2003;1007:232-237. 
The Journal of Pain Research is an international, peer reviewed, open access, online journal that welcomes laboratory and clinical findings in the fields of pain research and the prevention and management of pain. Original research, reviews, symposium reports, hypothesis formation and commentaries are all considered for publication
The manuscript management system is completely online and includes a very quick and fair peer-review system, which is all easy to use. Visit http://www.dovepress.com/testimonials.php to read real quotes from published authors.

Submit your manuscript here: https://www.dovepress.com/journal-of-pain-research-journal 